\title{
Batteriespeicher: Leistungsfähigkeit im Netzbetrieb
}

\author{
W. Vitovec, M. Wurm OVE
}

Online publiziert am 13. Februar 2019

(c) Springer-Verlag GmbH Austria, ein Teil von Springer Nature 2019

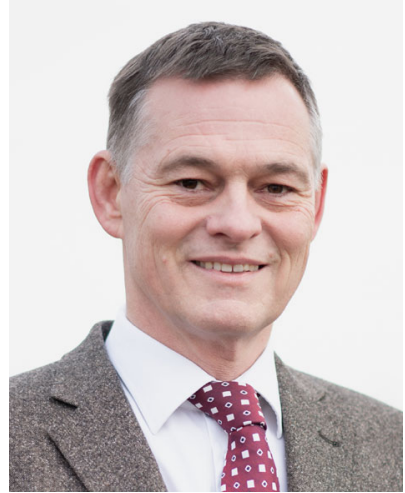

Dipl.-Ing. Dr. Wolfgang Vitovec

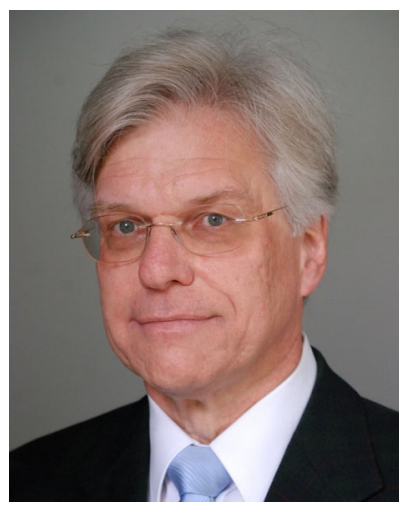

Dipl.-Ing. Dr. Manfred Wurm OVE

\author{
Liebe Leserin, lieber Le- \\ ser!
}

Die Erhöhung des Anteils an Strom aus erneuerbaren Energieträgern ist erklärtes Ziel der Politik, um die $\mathrm{CO}_{2}-$ Emissionen der Stromerzeugung zu senken, und dami eine wesentliche Maßnahme zum Klimaschutz. Doch die erneuerbaren Energieträger - speziell Sonne und Wind - unterliegen starken Schwankungen und stellen daher große Herausforderungen an eine stabile Stromversorgung dar. Batterien könnten zukünftig im Stromnetz einen wichtigen Beitrag zum Ausgleich von Erzeugung und Verbrauch, aber auch zur Stabilisierung leisten. Um diese Hypothese praktisch und auch theoretisch näher zu untersuchen, hat sich der Verteilnetzbetreiber Netz Niederösterreich $\mathrm{GmbH}$ dazu entschlossen, einen Li-lonen Groß-Batteriespeicher mit einer Leistung von 2,5 MVA und einer nutzbaren Kapazität von 2,2 MWh zu testen. Im Rahmen des vom Klimafonds der österreichischen Bundesregierung geförderten Forschungsprojektes "BatterieSTABIL" werden gemeinsam mit den Partnern Technische Universität Wien und Austrian Institute of Technology (AIT) Funktionen getestet, die essentielle Bestandteile einer zuverlässigen, sicheren und stabilen Stromversorgung sind.

Insbesondere sind dies System- und Netzdienstleistungen. Die Systemdienstleistungsprodukte wurden bisher maßgeblich aus Großkraftwerken (Wasserkraft, Kohle usw.) bereitgestellt. In den kommenden Jahren ist damit zu rechnen, dass vor allem konventionelle Großkraftwerke vom Netz gehen und die elektrische Leistung zunehmend aus volatilen erneuerbaren Energiequellen generiert werden wird. Dies erfordert für die Bereitstellung der Netz- und Systemdienstleistungen neue Ansätze, wobei Energiespeicher hier wesentliche Beiträge zu leisten haben werden. Von Batterien wird die Bereitstellung mehrerer Netzdienstleistungen gleichzeitig im sogenannten "multimodalen Betrieb" für verschiedene Stakeholder in der Energieversorgungskette als ein netztechnisch höchst nutzbringendes und auch wirtschaftlich sinnvolles Konzept angesehen. Als multimodaler Betrieb wird hier der Betrieb eines Batteriespeichers für die Erbringung verschiedener Netzdienstleistungen definiert, die auch kombiniert abgerufen werden können.

Das Forschungsvorhaben baut genau auf diesen Aspekten auf. Ein Ziel des Projektes ist es zu demonstrieren, wie zusätzlich zur Möglichkeit der Bereitstellung von etablierten Systemdienstleistungen (Primärregelleistung, Spannungshaltung und Versorgungswiederaufbau) das Potential des Batteriespeichers im multimodalen Betrieb durch Erbringung weiterer Systemdienstleistungen zur Systemstabilisierung (z. B. virtuelle Schwungmasse, dynamische Blindleistungskompensation und Spannungsregelung sowie symmetrierendes Verhalten) ausgeschöpft werden kann. Weiters wird die Fähigkeit zum Schwarzstart und für den Inselbetrieb untersucht.

Der erste Beitrag von Marchgraber, Alács, Gawlik beschäftigt sich mit dem Betrieb von Batteriespeichern zur Vorhaltung und Erbringung von Primärregelreserven samt dem dazu notwendigen Lademanagement. Es werden verschiedene Nachladestrategien gegenübergestellt, und der Einfluss verschiedener Auslegungsgrößen von Batteriespeichern auf die Ergebnisse wird untersucht. Weiters wird die Anpassung der regulatorischen Rahmenbedingungen zur Erbringung von Primärregelreserven hinsichtlich des Zugangs von Batteriespeichern zum Primärregelenergiemarkt betrachtet, um eine geeignete Auslegung von Batteriespeichern für zukünftige Regelleistungsprodukte frühzeitig zu fördern.

Im zweiten Beitrag von Jonke, Anta, Seitl wird eine Methode, der sogenannte Controller-Hardware-in-the-loop(c-HIL)-Ansatz, vorgestellt, um die Funktionalitäten eines Konverters für erneuerbare Energiesysteme zu überprüfen. Es wird gezeigt, wie hiermit sowohl die prinzipielle Funktion des Konverters als auch erweiterte Funktionen zur Unterstützung der Netzregelung, wie Q(U)-, P(f)Regelung usw., überprüft werden können. Im Rahmen der Untersuchungen dieses Beitrags wird ein Batteriespeichersystem mit der C-HIL-Methode analysiert und dabei mit realen Messdaten validiert.

Ein weiterer Beitrag berichtet über die Erkenntnisse von 110und 30-kV-Netzkurzschlussversuchen, die mit dem Batteriespeicher durchgeführt wurden. Da eine eingehende Analyse etwa der dynamischen Spannungsstützung bei tatsächlichen Netzfehlern aufgrund des messtechnischen Aufwands im praktischen Netzbetrieb so gut wie nie durchgeführt werden kann, wurde im Zuge der Inbetriebnahme des Batteriespeichers die Gelegenheit genutzt, um sowohl LVRT-Verhalten als auch dynamische Spannungsstützung bei realen symmetrischen und unsymmetrischen Netzkurzschlüssen zu untersuchen. Die Bewertung der maßgeblichen Messgrö-

Vitovec, Wolfgang, EVN AG, EVN-Platz 1, 2344 Maria Enzersdorf,

Österreich; Wurm, Manfred, Netz Niederösterreich GmbH, EVN-Platz 1

2344 Maria Enzersdorf, Österreich (E-Mail: manfred.wurm@netz-noe.at) 
Ben erfolgt in Hinblick auf die anzuwendenden Netzanschlussregeln.

Marchgraber, Gawlik, Wurm befassen sich abschließend mit der Modellierung von umrichterbasierten Einspeisungen in Kurzschlussberechnungen. In der aktualisierten Norm zur Kurzschlussrechnung IEC60909:-0:2016 wird eine Berücksichtigung dieser Anlagen ab einem Beitrag zum Kurzschlussstrom von $5 \%$ gefordert, worin der Kurzschlussbeitrag als Stromquelle im Mitsystem angenähert wird; das Verhalten im Gegensystem wird nicht näher definiert. Im Rahmen der vorliegenden Arbeit wird ein Modell vorgestellt, welches die Anforderungen gemäß VDE-AR-N-4110:2017-02 hinsichtlich des Kurzschlussverhaltens erfüllt. Die simulierten Rechenergeb- nisse des Modells werden mit Messergebnissen aus Netzkurzschlussversuchen verglichen.

Darüber hinaus findet sich in dieser Ausgabe noch ein Beitrag zum Thema Globale Erdungssysteme: Bestimmung der Erdungsspannung (EPR) in Kabelnetzen im einpoligen Fehlerfall. Fickert, Mallits, Resch untersuchen darin die maßgebenden Einflussfaktoren auf die Stromund Potentialverhältnisse in verbundenen (globalen) Erdungssystemen hinsichtlich der Sicherstellung der Personensicherheit und deren praktische Bedeutung im einpoligen Fehlerfall.

Der herzliche Dank gilt allen Autoren, die durch ihren Beitrag das Erscheinen dieses Themenhefts möglich gemacht haben! 